УДК 517.555

\author{
A. I. BANDURA, YA. I. SAVCHUK
}

\title{
STRUCTURE OF THE SET OF BOREL EXCEPTIONAL VECTORS FOR ENTIRE CURVES
}

\begin{abstract}
A. I. Bandura, Ya. I. Savchuk, Structure of the set of Borel exceptional vectors for entire curves, Mat. Stud. 53 (2020), 41-47.

It is proved that the structure of the set of Borel exceptional vectors for a transcendental entire curve is similar to that of the set of Nevanlinna deficient vectors for an entire curve of finite order. Particularly, any admissible system of Borel exceptional vectors for every $p$ dimensional transcendental entire curve with linearly independent components and without common zeros cannot have more than $p$ vectors.
\end{abstract}

In this paper, we use main results of theory of entire curves and notations from [1] and [5].

Let us remind these notations. Denote an entire curve $\vec{G}: \mathbb{C} \rightarrow \mathbb{C}^{p}$ as $\vec{G}(z)=\left(g_{1}(z)\right.$, $\left.g_{2}(z), \ldots, g_{p}(z)\right)$, where $g_{k}(z)$ is an entire function, $k \in\{1,2, \ldots, p\}$. Let us consider entire curves with linearly independent components and without common zeros. In other words, we assume that an entire curve $\vec{G}: \mathbb{C} \rightarrow \mathbb{C}^{p}$ has linearly independent components $g_{k}(z)$ and has no common zeros for all $g_{k}(z), k \in\{1,2, \ldots, p\}$.

For $\vec{a}=\left(a_{1}, a_{2}, \ldots, a_{p}\right) \in \mathbb{C}^{p}$ and $\vec{b}=\left(b_{1}, b_{2}, \ldots, b_{p}\right) \in \mathbb{C}^{p}$ the notation $\vec{a} \vec{b}$ means the dot product of these vectors, that is $\vec{a} \vec{b}=\sum_{j=1}^{p} a_{j} \bar{b}_{j}$, where $\bar{b}_{j}$ is complex conjugate to $b_{j}$.

For every $p$-dimensional vector $\vec{a}=\left(a_{1}, a_{2}, \ldots, a_{p}\right) \neq \overrightarrow{0}$ the dot product $\vec{G}(z) \vec{a}=$ $\sum_{k=1}^{p} g_{k}(z) \bar{a}_{k}$ is an entire function. Denote by $n(t, \vec{a}, \vec{G})$ the number of zeros of the dot product $\vec{G}(z) \vec{a}$ in the disc $\{z:|z| \leq t\}$, where each zero is counted according to its multiplicity. Every zero of the function $\vec{G}(z) \vec{a}$ is called $a$-point of the entire curve $\vec{G}(z)$. Let us denote

$$
N(r, \vec{a}, \vec{G})=\int_{0}^{r} \frac{n(t, \vec{a}, \vec{G})-n(0, \vec{a}, \vec{G})}{t} d t+n(0, \vec{a}, \vec{G}) \ln r
$$

where $n(0, \vec{a}, \vec{G})$ stands for the multiplicity of zero of the dot product $\vec{G}(z) \vec{a}$ at the point $z=0$.

The growth characteristic $T(r, \vec{G})$ is defined as following

$$
T(r, \vec{G})=\frac{1}{2 \pi} \int_{0}^{2 \pi} \ln \left\|\vec{G}\left(r e^{i \varphi}\right)\right\| d \varphi=\frac{1}{2 \pi} \int_{0}^{2 \pi} \ln \sqrt{\sum_{k=1}^{p}\left|g_{k}\left(r e^{i \varphi}\right)\right|^{2}} d \varphi
$$

2020 Mathematics Subject Classification: 30D35.

Keywords: entire curve; integral curve; Borel exceptional vector; Nevanlinna deficient vector; meromorphic function; admissible system of vectors.

doi:10.30970/ms.53.1.41-47

(C) A. I. Bandura, Ya. I. Savchuk, 2020 
We will use the definition of the growth category from [2, p. 44]. Let $\alpha(r)$ be a function defined for $r>0$, which is non-negative and non-decreasing for sufficiently large $r$ (if $\alpha(r)$ satisfies this condition, we write $\alpha(r) \in \Lambda$ ).

The number

$$
\rho=\rho[\alpha]=\varlimsup_{r \rightarrow+\infty} \frac{\ln ^{+} \alpha(r)}{\ln r}
$$

is called the order of $\alpha(r)$. The number

$$
\sigma=\sigma[\alpha]=\varlimsup_{r \rightarrow+\infty} \frac{\alpha(r)}{r^{\rho}}
$$

is called the magnitude of type of the function $\alpha(r)$. If $\sigma=0$, we say that $\alpha(r)$ has minimal type; if $0<\sigma<\infty$, we say that $\alpha(r)$ has normal (or mean) type; if $\sigma=\infty$, we say that $\alpha(r)$ has maximal type.

Let $\alpha(r)$ be a function of finite order $\rho$. We say that $\alpha(r)$ belongs to the convergence class or to the divergence class depending on whether the integral $\int_{1}^{\infty} \frac{\alpha(r)}{r^{\rho+1}} d r$ converges or diverges.

We say that functions $\alpha_{1}(r), \alpha_{2}(r) \in \Lambda$ are of the same growth category if they have the same order, and, if the order is finite, have the same type and either both belong to the convergence class, or both belong to the divergence class. We say that $\alpha_{2}(r)$ is of higher growth category than $\alpha_{1}(r)$ if one of the following conditions is satisfied:

1. $\rho\left[\alpha_{2}\right]>\rho\left[\alpha_{1}\right]$.

2. $\rho\left[\alpha_{1}\right]=\rho\left[\alpha_{2}\right]<\infty, \alpha_{1}(r)$ is of minimal type, and $\alpha_{2}(r)$ is of normal or maximal type.

3. $\rho\left[\alpha_{1}\right]=\rho\left[\alpha_{2}\right]<\infty, \alpha_{1}(r)$ is of normal type, and $\alpha_{2}(r)$ is of maximal type.

4. $\rho\left[\alpha_{1}\right]=\rho\left[\alpha_{2}\right]<\infty, \alpha_{1}(r)$ and $\alpha_{2}(r)$ are of minimal type, $\alpha_{1}(r)$ belongs to the convergence class, and $\alpha_{2}(r)$ belongs to the divergence class.

By analogy to a definition of a Borel exceptional value for a meromorphic function (see [2, p. 49]) a vector $\vec{a} \in \mathbb{C}^{p} \backslash\{\overrightarrow{0}\}$ is called a Borel exceptional vector of an entire curve $\vec{G}: \mathbb{C} \rightarrow \mathbb{C}^{p}$, if the growth category of $N(r, \vec{a}, \vec{G})$ is lower than the growth category of $T(r, \vec{G})$. In this paper, our main object of investigation is Borel exceptional vectors. Other kinds of exceptional and deficient vectors for entire curves were considered in $[9,10]$ (Valiron deficient vectors) [6,7] (Nevanlinna deficient vectors), [3] (averaged deficiency). More modern bibliography on this topic is listed in review paper of S. Mori [4].

It is known (for example, see [2, p. 98, Th. 2.2]), that any transcendental meromorphic function cannot have more than two Borel exceptional values.

We will generalize the result for the case of entire curves.

Let $L$ be some $q$-dimensional subspace in $\mathbb{C}^{p}$. We call [6] the system of vectors $M$ of $L$ admissible in $L$, if for card $M \leq q$ all vectors of $M$ are linearly independent and if for $\operatorname{card} M>q$ any $q$ vectors of $M$ are linearly independent.

Let the set $S$ lie in a $q$-dimensional subspace of the space $\mathbb{C}^{p}$ and contain $q$ linearly independent vectors. We call [6] the subset $M \subset S$ maximally admissible in $S$, if: a) any $q$ vectors of $M$ are linearly independent; b) any vector of $S \backslash M$ is a linear combination of some $q-1$ vectors of $M$.

Theorem 1. For every transcendental entire curve $\vec{G}: \mathbb{C} \rightarrow \mathbb{C}^{p}$ with linearly independent components and without common zeros any admissible system of Borel exceptional vectors cannot have more than $p$ vectors. 
Proof. Let $\vec{a}_{1}, \vec{a}_{2}, \ldots, \vec{a}_{k}$ be an admissible system of vectors in $\mathbb{C}^{p}$. By the second main theorem for entire curves one has

$$
(k-p) T(r, \vec{G}) \leq \sum_{j=1}^{k} N\left(r, \vec{a}_{j}, \vec{G}\right)+Q(r, \vec{G}),
$$

where $Q(r, \vec{G})$ is such a function that $Q(r, \vec{G})=O(\ln r)$ as $r \rightarrow \infty$, if $G$ has finite order. If $G$ has infinite order then $Q(r, \vec{G})=O(\ln T(r, \vec{G})+\ln r)$, as $r$ tends to $\infty$, outside some set of intervals on $[0, \infty)$, which has finite measure.

Assume that there exists a transcendental entire curve $\vec{G}$ having $(p+1)$ admissible Borel exceptional vectors $\vec{a}_{1}, \vec{a}_{2}, \ldots, \vec{a}_{p+1}$. Then (1) implies for $k=p+1$

$$
T(r, \vec{G}) \leq \sum_{j=1}^{p+1} N\left(r, \vec{a}_{j}, \vec{G}\right)+Q(r, \vec{G})
$$

If an entire curve $\vec{G}$ has infinite order then $N\left(r, \vec{a}_{j}, \vec{G}\right), j=1,2, \ldots, p+1$, have finite order. Therefore there exists $C<\infty$ such that for $r \geq r_{0}$ the following inequality

$$
\sum_{j=1}^{p+1} N\left(r, \vec{a}_{j}, \vec{G}\right) \leq r^{C}
$$

holds. Then outside some set $E$ of finite measure $L$ we have

$$
T(r, \vec{G}) \leq r^{C}+O\left(\ln T(r, \vec{G}+\ln r) \leq r^{C}+\frac{1}{2} T(r, \vec{G}),\right.
$$

whence $T(r, \vec{G}) \leq 2 r^{C}$. If $r \notin E$, then there exists a point $r^{\prime} \in[r, r+L+1]$ such that $r^{\prime} \notin E$. Thus,

$$
T(r, \vec{G}) \leq T\left(r^{\prime}, \vec{G}\right) \leq 2 r^{\prime C} \leq 2(r+L+1)^{C} \leq 3 r^{C},
$$

if $r$ is arbitrarily large. So, we conclude that $T(r, \vec{G})$ has finite order. It is a contradiction.

If an entire curve $\vec{G}$ has finite order then $Q(r, \vec{G})=o(T(r, \vec{G}))$, and (2) can be rewritten as follows:

$$
(1+o(1)) T(r, \vec{G}) \leq \sum_{j=1}^{p+1} N\left(r, \vec{a}_{j}, \vec{G}\right) .
$$

From this inequality it follows that if $\vec{G}$ has positive order, then all functions $N\left(r, \vec{a}_{j}, \vec{G}\right)$, $j=1,2, \ldots, p+1$, cannot have lower growth category than growth category of $T(r, \vec{G})$, i.e. we again obtain a contradiction.

If $\vec{G}$ has zero order then we consider the case, when $n\left(r, \vec{a}_{j}, \vec{G}\right)=O(1)$, i.e. $N\left(r, \vec{a}_{j}, \vec{G}\right)=$ $O(\ln r), j=1,2, \ldots, p+1$. Then from $(3)$ we obtain that $T(r, \vec{G})=O(\ln r)$, i.e. $G$ is not a transcendental entire curve and it contradicts hypothesis of the theorem.

The proved theorem does not give a complete description of the structure of the set of Borel exceptional vectors by analogy to description of structure of the set of Nevanlinna deficient vectors in [6]. For example, let us consider an entire curve

$$
\vec{G}(z)=\left(1, z, \ldots, z^{p-2}, e^{z}\right)
$$


Obviously, it has order one. So, $\ln r=o(T(r, \vec{G}))$. For any non-zero vector $\vec{a} \in B_{1}=\{\vec{b}=$ $\left.\left(b_{1}, b_{2}, \ldots, b_{p-1}, 0\right): b_{j} \in \mathbb{C}, j \in\{1,2, \ldots, p-1\}\right\}$ we have $n(r, \vec{a}, \vec{G})=O(1)$, i.e. $N(r, \vec{a}, \vec{G})=$ $O(\ln r)$. Therefore, all vectors from $B_{1} \backslash\{\overrightarrow{0}\}$ are Borel exceptional for the entire curve of (4). Also the vectors of the form $\vec{a}=(0,0, \ldots, 0, \alpha), \alpha \neq 0$ are Borel exceptional because for every $\vec{a}$ one has $n(r, \vec{a}, \vec{G})=0$. It means that $N(r, \vec{a}, \vec{G})=0$. One should observe that $B_{1}$ is a subspace of dimenstion $(p-1)$ in $\mathbb{C}^{p}$. Obviously, the system of $p$ vectors $\vec{e}_{1}=(1,0, \ldots, 0)$, $\vec{e}_{2}=(0,1,0, \ldots, 0), \ldots, \vec{e}_{p}=(0,0, \ldots, 0,1)$ is an orthonormal basis in $\mathbb{C}^{p}$ and they form an admissible system in $\mathbb{C}^{p}$. Every vector $\vec{e}_{j}$ is Borel exceptional for the curve $\vec{G}$ of form (4).

A set of all Borel exceptional vectors of entire curve $\vec{G}$ will denote by $\mathbf{B}(\vec{G})$. A main result of the paper is following

Theorem 2. For any transcendental entire curve $\vec{G}: \mathbb{C} \rightarrow \mathbb{C}^{p}$ with linearly independent components and without common zeros the set $\mathbf{B}(\vec{G}) \cup\{\overrightarrow{0}\}$ is a finite union of subspaces $A_{j} \subset \mathbb{C}^{p}$ of dimension $\leq p-1$. Moreover, there exist no more $p$ linearly independent vectors such that every $A_{j}$ is a linear span of these vectors.

Firstly, we will prove the following lemma.

Lemma 1. Let $\vec{G}: \mathbb{C} \rightarrow \mathbb{C}^{p}$ be an entire transcendental curve with linearly independent components and without common zeros, $q \leq p$ and $\vec{b}_{1}, \vec{b}_{2}, \ldots, \vec{b}_{q}$ is a system of linearly independent vectors of $\mathbf{B}(\vec{G}), B$ be the linear span of the vectors $\vec{b}_{1}, \vec{b}_{2}, \ldots, \vec{b}_{q}$; and $B_{1}, B_{2}$, $\ldots, B_{q}$ be the linear spans of the vector systems $\vec{b}_{2}, \vec{b}_{3}, \ldots, \vec{b}_{q}, \vec{b}_{1}, \vec{b}_{3}, \vec{b}_{4}, \ldots, \vec{b}_{q}, \ldots, \vec{b}_{1}, \vec{b}_{2}$, $\ldots, \vec{b}_{q-1}$, respectively. Then one has one of the following cases:

1. $B \subset \mathbf{B}(\vec{G}) \cup\{\overrightarrow{0}\}$;

2. $B \cap \mathbf{B}(\vec{G}) \subset \bigcup_{j=1}^{q} B_{j}$.

Proof. Let us consider a vector-valued function in $\mathbb{C}^{q}$

$$
\vec{G}_{1}(z)=\left(\vec{G}(z) \vec{b}_{1}, \vec{G}(z) \vec{b}_{2}, \ldots, \vec{G}(z) \vec{b}_{q}\right) \cdot \Phi(z)
$$

where $\Phi(z)$ is some meromorphic function in $\mathbb{C}$ without zeros and whose poles are common zeros of these functions $\vec{G}(z) \vec{b}, \vec{G}(z) \vec{b}_{2}, \ldots, \vec{G}(z) \vec{b}_{q}$. The components of $p$-dimensional entire curve $\vec{G}(z)$ are linearly independent by hypothesis of the theorem, the vectors $\vec{b}_{1}, \ldots, b_{q}$ are also linearly independent and $q \leq p$. Therefore, the functions $\vec{G}(z) \vec{b}_{1}, \ldots, \vec{G}(z) \vec{b}_{q}$ are linearly independent. Thus, the curve $\vec{G}_{1}(z)$ is a $q$-dimensional entire curve.

Clearly, that the growth category of $N(r, \Phi)$ is lower than the growth category of $T(r, \vec{G})$.

It is obvious that for any vector $\vec{\lambda}=\left(\bar{\lambda}_{1}, \ldots, \bar{\lambda}_{q}\right) \in \mathbb{C}^{p} \backslash\{\overrightarrow{0}\}$ and the vector $\vec{b}=\lambda_{1} \vec{b}_{1}+$ $\ldots+\lambda_{q} \vec{b}_{q}$ corresponding to it one has $\vec{G}(z) \vec{b}=\vec{G}_{1}(z) \vec{\lambda} / \Phi(z)$. It follows that

$$
N(r, \vec{b}, \vec{G})=N\left(r, \vec{\lambda}, \vec{G}_{1}\right)+N(r, \Phi) .
$$

We note that the growth category of $T\left(r, \vec{G}_{1}\right)$ cannot exceed growth category of $T(r, \vec{G})$, because

$$
T\left(r, \vec{G}_{1}\right)+N(r, \Phi)=\frac{1}{2 \pi} \int_{0}^{2 \pi} \ln \left\{\sum_{j=1}^{q}\left|\vec{G}\left(r e^{i \varphi}\right) \vec{b}_{j}\right|^{2}\right\}^{1 / 2}+O(1) \leq
$$




$$
\leq \frac{1}{2 \pi} \int_{0}^{2 \pi} \ln \left\{\sum_{j=\omega+1}^{q}\left\|\vec{G}\left(r e^{i \varphi}\right)\right\|^{2} \cdot\left\|\vec{b}_{j}\right\|^{2}\right\}^{1 / 2} d \varphi+O(1)=T(r, \vec{G})+O(1), \quad r \rightarrow \infty .
$$

There are two possible cases:

1. the growth category of $T\left(r, \vec{G}_{1}\right)$ is lower that the growth category of $T(r, \vec{G})$;

2. the growth category of $T\left(r, \vec{G}_{1}\right)$ is the same as the growth category of $T(r, \vec{G})$.

We will consider every case separately.

1) It is obvious that $\vec{b}_{1}, \vec{b}_{2}, \ldots, \vec{b}_{q}$ is a basis in $B$. Thus, any vector $\vec{b} \in B \backslash\{\overrightarrow{0}\}$ admits a representation $\vec{b}=\lambda_{1} \vec{b}_{1}+\ldots+\lambda_{q} \vec{b}_{q}$. Therefore, we put $\vec{\lambda}=\left(\bar{\lambda}_{1}, \ldots, \bar{\lambda}_{q}\right)$. Equality (5) implies that $N(r, \vec{b}, \vec{G})$ has lower growth category than $T(r, \vec{G})$, because $N\left(r, \vec{\lambda}, \vec{G}_{1}\right) \leq T\left(r, \vec{G}_{1}\right)+$ $O(1)$. Hence, $B \subset \mathbf{B}(\vec{G}) \cup\{\overrightarrow{0}\}$.

2) Suppose that there exists $\vec{b}_{0}=\lambda_{01} \vec{b}_{1}+\ldots+\lambda_{0 q} \vec{b}_{q} \in B \cap \mathbf{B}(\vec{G}), \vec{b}_{0} \notin \bigcup_{j=1}^{q} B_{j}$. Obviously, that the system of vectors

$$
\vec{\lambda}^{(0)}=\left(\bar{\lambda}_{01}, \ldots, \bar{\lambda}_{0 q}\right), \vec{\lambda}^{(1)}=(1,0, \ldots, 0), \vec{\lambda}^{(2)}=(0,1,0, \ldots, 0), \quad \vec{\lambda}^{(q)}=(0, \ldots, 0,1)
$$

is admissible in $\mathbb{C}^{q}$. In view of equality (5), we also have $N\left(r, \vec{b}_{j}, \vec{G}\right)=N\left(r, \vec{\lambda}^{(j)}, \vec{G}_{1}\right)+N(r, \Phi)$, $j=0,1, \ldots, q$. Thus, the growth category of every function $N\left(r, \vec{\lambda}^{(j)}, \vec{G}_{1}\right)$ is lower than the growth category of $T(r, \vec{G})$, whence it is also lower than the growth category of $T\left(r, \vec{G}_{1}\right)$. It means that all vectors $\vec{\lambda}^{(0)}, \vec{\lambda}^{(1)}, \ldots, \vec{\lambda}^{(q)}$ are Borel exceptional for $\vec{G}_{1}$, but by Theorem 1 it is impossible. The obtained contradiction proves the statement of the lemma.

Proof of Theorem 2. Choose a maximally admissible system of Borel exceptional vectors (see also [6]) in $\mathbb{C}^{p}$ of the given entire curve. By Theorem 1 there exist at most $p$ such vectors. Denote these vectors by

$$
\vec{a}_{1}, \vec{a}_{2}, \ldots, \vec{a}_{k}
$$

We note that for all $j=1, \ldots, k$ any non-zero vector of one-dimensional space $L_{j}=$ $\left\{\alpha \vec{a}_{1}: \alpha \in \mathbb{C}\right\}$ is Borel exceptional for $\vec{G}$. Hence, $L_{j} \subset \mathbf{B}(\vec{G}) \cup\{\overrightarrow{0}\}$.

Let $A$ be the linear span of $\vec{a}_{1}, \vec{a}_{2}, \ldots, \vec{a}_{k}$. Clearly, $\mathbf{B}(\vec{G}) \subset A$. By Lemma 1 one of two cases is possible.

1) $A \subset \mathbf{B}(\vec{G}) \cup\{\overrightarrow{0}\}$, i. e. $A=\mathbf{B}(\vec{G}) \cup\{\overrightarrow{0}\}$. It proves the statement of the theorem. Obviously, this case is possible if $k<n$.

2) $A \cap \mathbf{B}(\vec{G})=\mathbf{B}(\vec{G}) \subset \bigcup_{j=1}^{k} A^{(j)}$, that is $\mathbf{B}(\vec{G})=\bigcup_{j=1}^{k}\left(\mathbf{B}(\vec{G}) \cap A^{(j)}\right)$, where $A^{(1)}, A^{(2)}$, $\ldots, A^{(k)}$ are subspaces of dimension $(k-1)$. Every such a subspace is generated as linear spans of all collections of $(k-1)$ vectors with $(6)$.

For $A^{(j)}$, for which $\mathbf{B}(\vec{G}) \cap A^{(j)} \neq A^{(j)} \backslash\{\overrightarrow{0}\}$, we carry out the arguments given above with $A$, and obtain $\mathbf{B}(\vec{G}) \cap A^{(j)}=\bigcup_{s=1}^{k-1}\left(\mathbf{B}(\vec{G}) \cap A^{(j s)}\right)$, where $A^{(j s)}$ are subspaces of dimension $(k-2)$, which are obtained from $A^{(j)}$ in the same way that $A^{(j)}$ was obtained from $A$.

At the next stage for such $A^{(j s)}$ with $\mathbf{B}(\vec{G}) \cap A^{(j s)} \neq A^{(j s)} \backslash\{\overrightarrow{0}\}$ we find $\mathbf{B}(\vec{G}) \cap A^{(j s)}=$ $\bigcup_{i=1}^{k-2}\left(\mathbf{B}(\vec{G}) \cap A^{(j s i)}\right)$, where $A^{(j s i)}$ are subspaces of dimension $(k-3)$.

Finally, in no more than $(k-1)$-th stage we get $\mathbf{B}(\vec{G})=\bigcup_{j}\left(\mathbf{B}(\vec{G}) \cap A_{j}\right)$, whereby $A_{j}$ we denote those $A^{(j)}, A^{(j s)}, A^{(j s i)}, \ldots$, which in view of above given remark are contained in $\mathbf{B}(\vec{G}) \cup\{\overrightarrow{0}\}$. Hence, $\mathbf{B}(\vec{G}) \cap A_{j}=A_{j} \backslash\{\overrightarrow{0}\}$. Therefore, $\mathbf{B}(\vec{G}) \cup\{\overrightarrow{0}\}=\bigcup_{j} A_{j}$. 
It is known (for example, see [2, p. 114, Th.1.1]) that any transcendental meromorphic function of non-integer or zero order cannot have more than one Borel exceptional value. The property was generalized for case of entire curves.

Theorem 3. Any transcendental entire curve $\vec{G}: \mathbb{C} \rightarrow \mathbb{C}^{p}$ of non-integer or zero order cannot have more than $(p-1)$ linearly independent Borel exceptional vectors.

Proof. Assume the contrary, that is, there exists an entire transcendental curve $\vec{G}(z)=$ $\left(g_{1}(z), g_{2}(z), \ldots, g_{p}(z)\right)$ of non-integer or zero order, which has $p$ linearly independent Borel exceptional vectors. We denote these vectors by $\vec{a}_{1}, \vec{a}_{2}, \ldots, \vec{a}_{p}$. Obviously, the entire functions $\widetilde{g}_{1}(z)=\vec{G}(z) \vec{a}_{1}, \widetilde{g}_{2}(z)=\vec{G}(z) \vec{a}_{2}, \ldots, \widetilde{g}_{p}(z)=\vec{G}(z) \vec{a}_{p}$ are linearly independent and have no common zeros. Let us consider an entire curve $\vec{G}_{p}(z)=\left(\widetilde{g}_{1}(z), \widetilde{g}_{2}(z), \ldots, \widetilde{g}_{p}(z)\right)$. It is easy to check that

$$
T\left(r, \vec{G}_{p}\right)=T(r, \vec{G})+O(1) .
$$

Clearly that $N\left(r, \vec{a}_{j}, \vec{G}\right)=N\left(r, \vec{e}_{j} \vec{G}_{p}\right)=N\left(r, 0, \widetilde{g}_{j}\right), j=1,2, \ldots, n$. Taking into account (7), the vectors $\vec{e}_{1}=(1,0, \ldots, 0), \vec{e}_{2}=(0,1,0, \ldots, 0), \ldots, \vec{e}_{p}=(0,0, \ldots, 0,1)$ are Borel exceptional for $\vec{G}_{p}$. From the obvious inequality $\ln \left(\alpha_{1}+\alpha_{2}+\ldots+\alpha_{n}\right) \leq \ln ^{+} \alpha_{1}+\ln ^{+} \alpha_{2}+$ $\ldots+\ln ^{+} \alpha_{n}+\ln n$ for arbitrary positive numbers $\alpha_{1}, \alpha_{2}, \ldots, \alpha_{n}$ we easy deduce

$$
\begin{gathered}
T\left(r, \vec{G}_{p}\right)=\frac{1}{2 \pi} \int_{0}^{2 \pi} \ln \left\|\vec{G}_{p}\left(r e^{i \varphi}\right)\right\| d \varphi= \\
=\frac{1}{2 \pi} \int_{0}^{2 \pi} \ln \left(\left|\widetilde{g}_{1}\left(r e^{i \varphi}\right)\right|^{2}+\left|\widetilde{g}_{2}\left(r e^{i \varphi}\right)\right|^{2}+\ldots+\left|\widetilde{g}_{p}\left(r e^{i \varphi}\right)\right|^{2}\right)^{1 / 2 d} d \varphi \leq \\
\leq \frac{1}{2 \pi} \int_{0}^{2 \pi} \ln \left(\left|\widetilde{g}_{1}\left(r e^{i \varphi}\right)\right|+\left|\widetilde{g}_{2}\left(r e^{i \varphi}\right)\right|+\ldots+\left|\widetilde{g}_{p}\left(r e^{i \varphi}\right)\right|\right) d \varphi= \\
\leq \frac{1}{2 \pi} \int_{0}^{2 \pi} \ln \left|\widetilde{g}_{1}\left(r e^{i \varphi}\right)\right| d \varphi+\frac{1}{2 \pi} \int_{0}^{2 \pi} \ln \left(1+\frac{\left|\widetilde{g}_{2}\left(r e^{i \varphi}\right)\right|}{\left|\widetilde{g}_{1}\left(r e^{i \varphi}\right)\right|}+\ldots+\frac{\left|\widetilde{g}_{p}\left(r e^{i \varphi}\right)\right|}{\left|\widetilde{g}_{1}\left(r e^{i \varphi}\right)\right|}\right) d \varphi \leq \\
=N(r, 0, \widetilde{g})+m\left(r, \frac{1}{\int_{0}}\left|\widetilde{g}_{1}\left(r e^{i \varphi}\right)\right| d \varphi+\frac{1}{2 \pi} \int_{0}^{2 \pi} \ln +\frac{\left|\widetilde{g}_{2}\left(r e^{i \varphi}\right)\right|}{\left|\widetilde{g}_{1}\left(r e^{i \varphi}\right)\right|} d \varphi+\ldots+m(r) \frac{\widetilde{g}_{p}}{\widetilde{g}_{1}}\right)+O(1) .
\end{gathered}
$$

Here we used Jensen's formula ([2, p. 13]). The functions $\widetilde{g}_{1}(z), \widetilde{g}_{2}(z), \ldots, \widetilde{g}_{p}(z)$ have no common zeros. Therefore,

$$
N\left(r, 0, \widetilde{g}_{1}\right) \leq N\left(r, \frac{\widetilde{g}_{2}}{\widetilde{g}_{1}}\right)+\ldots+N\left(r, \frac{\widetilde{g}_{p}}{\widetilde{g}_{1}}\right) .
$$

Then (8) yields

$$
T\left(r, \vec{G}_{p}\right) \leq \sum_{j=2}^{p}\left(m\left(r, \frac{\widetilde{g}_{j}}{\widetilde{g}_{1}}\right)+N\left(r, \frac{\widetilde{g}_{j}}{\widetilde{g}_{1}}\right)\right)+O(1)=\sum_{j=2}^{p} T\left(r, \frac{\widetilde{g}_{j}}{\widetilde{g}_{1}}\right)+O(1) .
$$

This inequality implies that at least one of the functions $T\left(r, \frac{\widetilde{g}_{j}}{\widetilde{g}_{1}}\right)$ (for example, $T\left(r, \frac{\widetilde{g}_{k}}{\widetilde{g}_{1}}\right)$ ) has the growth category which is not lower than $T\left(r, \vec{G}_{p}\right)$. From the other hand (see [5, p. 7]), $T\left(r, \frac{\widetilde{g}_{k}}{\widetilde{g}_{1}}\right) \leq T\left(r, \vec{G}_{p}\right)+C$. Thus, $T\left(r, \frac{\widetilde{g}_{k}}{\widetilde{g}_{1}}\right)$ has the same growth category as $T\left(r, \vec{G}_{p}\right)$. In 
particular, the meromorphic function $\frac{\widetilde{g}_{k}}{\widetilde{g}_{1}}$ has non-integer or zero order. Above we noted that all functions $N\left(r, 0, \widetilde{g}_{j}\right)$ have lower growth category than $T\left(r, \vec{G}_{p}\right)$. Since $N\left(r, \widetilde{g}_{k}\right) \leq N\left(r, 0, \widetilde{g}_{1}\right)$ and $N\left(r, 0, \frac{\widetilde{g}_{k}}{\widetilde{g}_{1}}\right) \leq N\left(r, 0, \widetilde{g}_{k}\right)$, the values 0 and $\infty$ are Borel exceptional values of $\frac{\widetilde{g}_{k}}{\widetilde{g}_{1}}$, and it is impossible. This contradiction proves the theorem.

Let us consider entire curve $\vec{G}(z)=\left(1, z, \ldots, z^{n-2}, f(z)\right)$, where $f(z)$ is an entire transcendental function of non-integer or zero order. It is easy to check that $T(r, \vec{G})=T(r, f)+$ $O(\ln r)$. Thus, $\vec{G}$ has the same order as $f$, and $\ln r=o(T(r, \vec{G}))$. Obviously, every with $(p-1)$ linearly independent vectors $\vec{e}_{1}=(1,0, \ldots, 0), \vec{e}_{2}=(0,1,0, \ldots, 0), \ldots, \vec{e}_{p-1}=$ $(0,0, \ldots, 0,1,0)$ is Borel exceptional for entire curve of form $\vec{G}(z)=\left(1, z, \ldots, z^{n-2}, f(z)\right)$.

The example demonstrates that Theorem 3 cannot be improved.

Finally, we pose the following open problem.

Problem 1. Is it possible to solve the inverse problem for the set of Borel exceptional vectors of entire curve by analogy to [7,8], where the problem is solved for the set of Nevanlinna deficient vectors?

\section{REFERENCES}

1. Gol'dberg A.A., Some questions of the theory of distribution of values of meromorphic functions, in: G. Wittich, Latest Investigations on Single-Valued Analytic Functions, Fizmatgiz, Moscow (1960). (in Russian)

2. Gol'dberg A.A., Ostrovskii I.V., Value distribution of meromorphic functions, Providence: AMS, 2008. (Translated from Russian ed. Moscow, Nauka, 1970).

3. Dektyarev I.M., Averaged deficiencies of holomorphic curves and divisors with an excessive deficiency value, Russian Mathematical Surveys, 44 (1989), №1, 237-238. doi: 10.1070/RM1989v044n01ABEH002015

4. Mori S., Topics on meromorphic mappings and defects, Complex Var. Elliptic Equ., 56 (2011), №1-4, 363-373. doi: 10.1080/17476930903394903

5. Petrenko V.P., Entire curves, Kharkiv: Vyshcha shkola, 1984. (in Russian)

6. Savchuk Ya.I., Structure of the set of defect vectors of entire and analytic curves of finite order, Ukr. Math. J., 37 (1985), №5, 494-499. doi: 10.1007/BF01061174

7. Savchuk Ya.I., Set of deficient vectors of integral curves, Ukr. Math. J., 35 (1983), №3, 334-338. doi: $10.1007 /$ BF01092190

8. Savchuk Ya.I., Inverse problem of the theory of distribution of the values of entire and analytic curves, Journal of Soviet Mathematics, 48 (1990), №2, 220-231. doi: 10.1007/BF01095801

9. Savchuk Ya.I., Valiron deficient vectors of entire curves of finite order, Journal of Soviet Mathematics, 52 (1990), №5, 3435-3437. doi: 10.1007/BF01099913

10. Toda N., Holomorphic curves with an infinite number of deficiencies, Proc. Japan Acad. Ser. A Math. Sci., 80 (2004), №6, 90-95. doi: 10.3792/pjaa.80.90.

Department of Advanced Mathematics

Ivano-Frankivsk National Technical University of Oil and Gas

Ivano-Frankivsk, Ukraine

andriykopanytsia@gmail.com

math@nung.edu.ua 